\title{
The transformation of an interfacial solitary wave of elevation at a bottom step
}

\author{
V. Maderich $^{1}$, T. Talipova ${ }^{2}$, R. Grimshaw ${ }^{3}$, E. Pelinovsky ${ }^{2,3}$, B. H. Choi ${ }^{4}$, I. Brovchenko ${ }^{1}$, K. Terletska ${ }^{1}$, and \\ D. C. Kim $^{4}$ \\ ${ }^{1}$ Department of Marine and River Systems, Institute of Mathematical Machine and System Problems, Kiev, Ukraine \\ ${ }^{2}$ Department of Nonlinear Geophysical Processes, Institute of Applied Physics, Nizhny Novgorod, Russia \\ ${ }^{3}$ Department of Mathematical Sciences, Loughborough University, Loughborough, UK \\ ${ }^{4}$ Department of Civil and Environmental Engineering, Sungkyunwan University, Suwon, Korea
}

Received: 3 November 2008 - Revised: 14 January 2009 - Accepted: 14 January 2009 - Published: 5 February 2009

\begin{abstract}
In this paper we study the transformation of an internal solitary wave at a bottom step in the framework of two-layer flow, for the case when the interface lies close to the bottom, and so the solitary waves are elevation waves. The outcome is the formation of solitary waves and dispersive wave trains in both the reflected and transmitted fields. We use a two-pronged approach, based on numerical simulations of the fully nonlinear equations using a version of the Princeton Ocean Model on the one hand, and a theoretical and numerical study of the Gardner equation on the other hand. In the numerical experiments, the ratio of the initial wave amplitude to the layer thickness is varied up onehalf, and nonlinear effects are then essential. In general, the characteristics of the generated solitary waves obtained in the fully nonlinear simulations are in reasonable agreement with the predictions of our theoretical model, which is based on matching linear shallow-water theory in the vicinity of a step with solutions of the Gardner equation for waves far from the step.
\end{abstract}

\section{Introduction}

Internal solitary waves (solitons) are commonly observed on ocean shelves, and their role in mixing and sediment transport has been intensively studied (Bogucki and Redekopp, 1999; Ribbe and Holloway, 2001; Stastna and Lamb, 2008). Observations show that there is a wide variety of processes

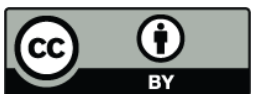

Correspondence to: R. Grimshaw (r.h.j.grimshaw@lboro.ac.uk) for the transformation of a nonlinear wave in the shelf zone due to changes in the depth and the background density stratification, including soliton fission (Zheng et al., 2001; Zhao et al., 2003; Orr and Mignerey, 2003; Helfrich, 1992; Vlasenko et al., 2005; Bourgault et al., 2007). Theoretically such processes have been well studied for a smoothly and slowly varying background when the Korteweg-de Vries equation and its modifications can be applied (Djordevic and Redekopp, 1978, Helfrich and Melville, 1986, Holloway et al., 1997, 1999; Zheng et al., 2001; Grimshaw et al., 2004, 2007). Such processes for internal waves in a basin of variable depth have also been studied numerically in the framework of fully nonlinear equations (Lamb, 2002, 2003; Vlasenko et al., 2005; Vlasenko and Stashchuk, 2007).

Recently, processes of solitary wave transformation have been explored for rapidly varying bottom topography. One such example is the shelf between Taiwan and Dongsha Islands which includes very steep areas when the bottom slope is 0.25 , while the solitary wavelength is comparable with the size of the area (Ramp et al., 2004). In the laboratory, interfacial solitary wave transformation on a sloping wall with slopes varying from $30^{\circ}$ to $130^{\circ}$ has been studied (Chen et al., 2007a, c). Theoretically, solitary wave transformation in a two-layer flow with a bottom step has been studied in the framework of the Korteweg-de Vries and Gardner equations, an extended version of the Korteweg-de Vries equation which includes both quadratic and cubic nonlinearity, (Grimshaw et al., 2008), and in a Boussinesq-like system for a two-layer flow with rapidly varying bottom topography (De Zarate and Nashbin, 2008).

The goal of this present paper is to study fission of an interfacial solitary wave at a bottom step for solitary waves of

Published by Copernicus Publications on behalf of the European Geosciences Union and the American Geophysical Union. 


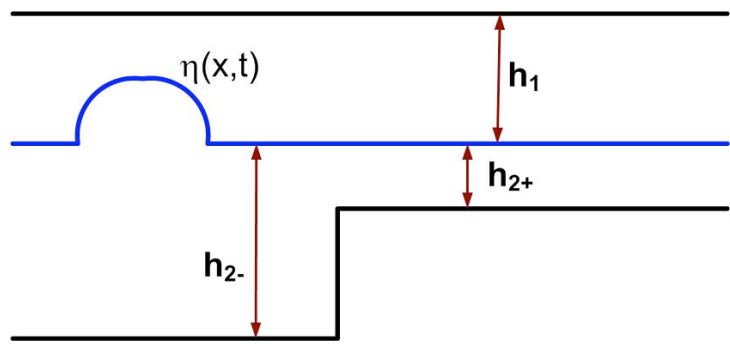

Fig. 1. Problem configuration.

moderate amplitudes (the ratio of the initial wave amplitude to the layer thickness is varied up to 0.5 ). The weakly nonlinear theory of such wave transformation at a step was developed by Grimshaw et al. (2008), and is briefly reproduced in Sect. 2, where we present some formulas for the solitary waves of moderate amplitude obtained in the framework of the Gardner equation). The applicability of the Kortewegde Vries and Gardner equations to model interface solitary waves is discussed in this section. Then we use a numerical model based on the Princeton Ocean Model (POM) (see Kanarska and Maderich, 2003; Brovchenko et al., 2007), which is briefly described in Sect. 3. This numerical model is applied here to a typical laboratory situation, in which a twodimensional fluid is stratified by salinity; two layers of different salinities are separated by a very narrow layer with a continuously varying salinity. The results of our numerical simulations for the transformation of a solitary wave at a step are discussed in Sects. 4 and 5 for waves of moderate and large amplitudes. The amplitude of the initial solitary wave of elevation in the computations is 1 and $4 \mathrm{~cm}$, while the thickness of the lower layer is $8 \mathrm{~cm}$, and after the step the thickness is $4 \mathrm{~cm}$; hence nonlinear effects for such waves are essential. The numerical results are then compared with theoretical predictions based on the Korteweg-de Vries and Gardner equations. Our results are summarized in the conclusion.

\section{Theoretical model}

The configuration is shown in Fig. 1, where the upper (lower) layer has density $\rho_{1}\left(\rho_{2}\right)$. An interfacial solitary wave approaches the bottom step from the right, and the water depth is then decreased. The solitary wavelength is assumed to be always larger than the water depth but for convenience we will say that the wave approaches from deep to shallow water.

If the solitary wave has a small amplitude, the process of its transformation in the vicinity of the step can be described by linear long-wave theory. This assumption was used by Grimshaw et al. (2008) who derived the following expressions for coefficients of wave reflection $R$ and transmission $T$ at the step,
$R=\frac{1-\frac{c_{+}}{c_{-}}}{1+\frac{c_{+}}{c-}}, \quad T=\frac{2}{1+\frac{c_{+}}{c_{-}}}$,

where $c_{ \pm}$is the speed of linear long interfacial waves in the deep (-) and shallow (+) parts of the water basin,

$c_{ \pm}=\sqrt{g \frac{\Delta \rho}{\rho} \frac{h_{1} h_{ \pm}}{h_{1}+h_{ \pm}}}$.

$\Delta \rho / \rho$ is the relative density jump, $g$ is the acceleration due to gravity, $h_{1}$ is thickness of upper layer, $h_{ \pm}$are the thicknesses of lower layers $h_{2 \pm}$ (the index 2 is omitted here and hereafter) in the deep (-) and shallow $(+)$ parts of the water basin. In fact, the applicability of these linear formulas to the transformation of nonlinear waves is not self-evident. It has been the subject of many special experimental and numerical studies for surface wave transformation at a step (Seabra-Santos et al., 1987; Liu and Cheng, 2001; Chang et al., 2001; Lin, 2004), and as a result, it has been concluded that formulas such as (1) are a very good description of the transformation for waves of moderate amplitude (the ratio amplitude/depth up to 0.4). We expect that this conclusion is valid also for interfacial solitary waves.

Far from the step (left or right), the wave propagates in a basin of constant depth and its unidirectional propagation can be described by the Korteweg- de Vries (KdV) equation if the wave amplitude is weak,

$\frac{\partial \eta}{\partial t}+(c+\alpha \eta) \frac{\partial \eta}{\partial x}+\beta \frac{\partial^{3} \eta}{\partial x^{3}}=0$,

where the dispersive and nonlinear coefficients are (in the Boussinesq approximation when $\Delta \rho / \rho \ll 1$ ),

$\beta=\frac{c h_{1} h_{2}}{6}, \quad \alpha=\frac{3 c}{2} \frac{h_{1}-h_{2}}{h_{1} h_{2}}$.

The steady-state solution of (3) is the KdV soliton,

$\eta=A \operatorname{sech}^{2}\left[\sqrt{\frac{3 A}{4} \frac{\left(h_{1}-h_{2}\right)}{h_{1}^{2} h_{2}^{2}}}(x-(c+\alpha A / 3) t)\right]$.

The amplitude is positive (elevation wave) if $h_{1}-h_{2}>0$ $(\alpha>0)$, and negative (depression wave) if $\left(h_{1}-h_{2}\right)<0$ $(\alpha<0)$. If $h_{1}-h_{2}=0$, as is well-known, interfacial solitons do not exist. We assume that the incident solitary wave propagates from the right towards the step, and hence $h_{2}=h_{-}$. In the vicinity of the step, the reflected and transmitted waves have the $\mathrm{KdV}$ soliton-like shapes, but their parameters do not satisfy the steady-state soliton solution (5),

$$
\begin{aligned}
& \eta_{\text {ref }}=A_{\text {ref }} \operatorname{sech}^{2}\left[\sqrt{\frac{3 A}{4} \frac{\left(h_{1}-h_{-}\right)}{h_{1}^{2} h_{-}^{2}} x}\right], \quad A_{\text {ref }}=R A, \\
& \eta_{t r}=A_{t r} \operatorname{sech}^{2}\left[\sqrt{\frac{3 A}{4} \frac{\left(h_{1}-h_{-}\right)}{h_{1}^{2} h_{-}^{2}}} \frac{c_{-}}{c_{+}} x\right], \quad A_{t r}=T A .
\end{aligned}
$$


Then for wave propagation away from the step, a soliton-like disturbance evolves in general into solitons and a dispersive wave train. The parameters of these secondary solitons are calculated in Grimshaw et al. (2008) using the rigorous theory of the $\mathrm{KdV}$ equation. In particular, only one soliton is formed in the reflected wave with amplitude

$$
\frac{A_{s r}}{A}=\left[\sqrt{2 R+\frac{1}{4}}-\frac{1}{2}\right]^{2} \text {. }
$$

The dynamics of the transmitted wave depends on the sign of the quadratic nonlinear term after the step. If the coefficient of the nonlinear term changes its sign after the step, the initial soliton-like disturbance is completely destroyed and transforms into radiation. Here we consider the situation when the sign of the nonlinearity is not changed (this requires that $h_{2}<h_{1}$ everywhere), and so the transmitted wave is transformed into secondary solitons (soliton fission) and their amplitudes are

$$
\begin{aligned}
& \frac{A_{s t r}^{m}}{A}=\left(\frac{c_{-}}{c_{+}}\right)^{2}\left(\frac{\alpha_{-}}{\alpha_{+}}\right)\left(\frac{\beta_{+}}{\beta_{-}}\right) \\
& {\left[\sqrt{2 T\left(\frac{c_{+}}{c_{-}}\right)^{2}\left(\frac{\alpha_{+}}{\alpha_{-}}\right)\left(\frac{\beta_{-}}{\beta_{+}}\right)+\frac{1}{4}}-\left(m+\frac{1}{2}\right)\right]^{2}}
\end{aligned}
$$

where $m=0,1,2, \ldots N-1$, and $N$ is the number of transmitted solitons,

$N=\left[\sqrt{2 T\left(\frac{c_{+}}{c_{-}}\right)^{2}\left(\frac{\alpha_{+}}{\alpha_{-}}\right)\left(\frac{\beta_{-}}{\beta_{+}}\right)+\frac{1}{4}}+\frac{1}{2}\right]$.

The values of $\alpha_{ \pm}$and $\beta_{ \pm}$are the nonlinear and dispersive coefficients at the different sides of step. The analysis of these secondary soliton parameters for various conditions based on Eqs. (8)-(10) is described in Grimshaw et al. (2008).

The KdV equation is valid for interfacial waves of weak amplitude. With an increase in amplitude cubic nonlinear effects become essential, and an extended version of the KdV equation should be used, Grimshaw et al. (2002b). If the interface lies approximately at the middle depth, it can be reduced to the Gardner equation which includes only the cubic nonlinear term when compared with KdV equation,

$$
\frac{\partial \eta}{\partial t}+\left(\alpha \eta+\alpha_{1} \eta^{2}\right) \frac{\partial \eta}{\partial x}+\beta \frac{\partial^{3} \eta}{\partial x^{3}}=0
$$

Here $\alpha_{1}$ is the cubic nonlinear coefficient which is always negative for interfacial waves (Kakutani and Yamasaki, 1978)

$\alpha_{1}=-\frac{3 c}{8 h_{1}^{2} h_{2}^{2}}\left(h_{1}^{2}+h_{2}^{2}+6 h_{1} h_{2}\right)$.

The Gardner equation like the KdV equation is fully integrable. Steady-state solitary wave solution of the Gardner equation can be found explicitly (see for instance Grimshaw et al., 2004, 2007),

$$
\begin{aligned}
& \eta(x, t)=\frac{D}{1+B \cosh (\gamma(x-V t)),} \\
& D=\frac{6 \beta \gamma^{2}}{\alpha}, B^{2}=1+\frac{6 \alpha_{1} \beta \gamma^{2}}{\alpha^{2}}, \quad V=\beta \gamma^{2},
\end{aligned}
$$

where $\gamma$ is a parameter characterizing the inverse width of the soliton. The soliton amplitude is

$A=\frac{D}{1+B}$,

and its sign coincides with the sign of the coefficient of quadratic nonlinearity $\alpha$. For interfacial waves the parameter $B$ varies between 0 and 1 . The soliton amplitude varies from small values, when the Gardner soliton (13) coincides with the $\mathrm{KdV}$ soliton (5), to the limiting value

$A_{\lim }=\frac{\alpha}{\left|\alpha_{1}\right|}$,

when the soliton has a "table-top" shape.

As has been pointed out previously for surface waves of moderate amplitude, the linear formulas for wave transformation at a step give a correct estimation of reflected and transmitted waves (Seabra-Santos et al., 1987; Liu and Cheng, 2001; Chang et al., 2001; Lin, 2004). We assume that the same result holds for interfacial waves of moderate amplitudes. With these assumptions the wave shape for transmitted and reflected waves in the vicinity of a step has the Gardner-soliton shape, but their parameters do not satisfy the soliton conditions,

$$
\begin{aligned}
& \eta_{t r}(x)=\frac{D T}{1+B \cosh \left(\frac{c_{-}}{c_{+}} \gamma x\right)}, \quad D=\frac{6 \beta_{-} \gamma^{2}}{\alpha_{-}}, \\
& B^{2}=1+\frac{6 \alpha_{1}-\beta-\gamma^{2}}{\alpha-^{2}} . \\
& \eta_{\text {ref }}(x)=\frac{D R}{1+B \cosh (\gamma x)}
\end{aligned}
$$

The number and amplitudes of the secondary solitons in the framework of the Gardner equation can in principle be found using the inverse scattering technique. However, simple analytical expressions such as Eqs. (8)-(10) are not available for the Gardner equation, and instead we will find them numerically.

For applications and comparison with the numerical results of the fully nonlinear system, the nonlinear effects need to be characterized quantitatively. In the Gardner equation nonlinearity may be characterized by a parameter $\varepsilon$,

$\varepsilon=\varepsilon_{q}+\varepsilon_{c}=\frac{\alpha A}{c}+\frac{\alpha_{1} A^{2}}{c}$,

where $A$ is the wave amplitude; it represents the sum of the quadratic and cubic nonlinear terms. For weakly nonlinear 


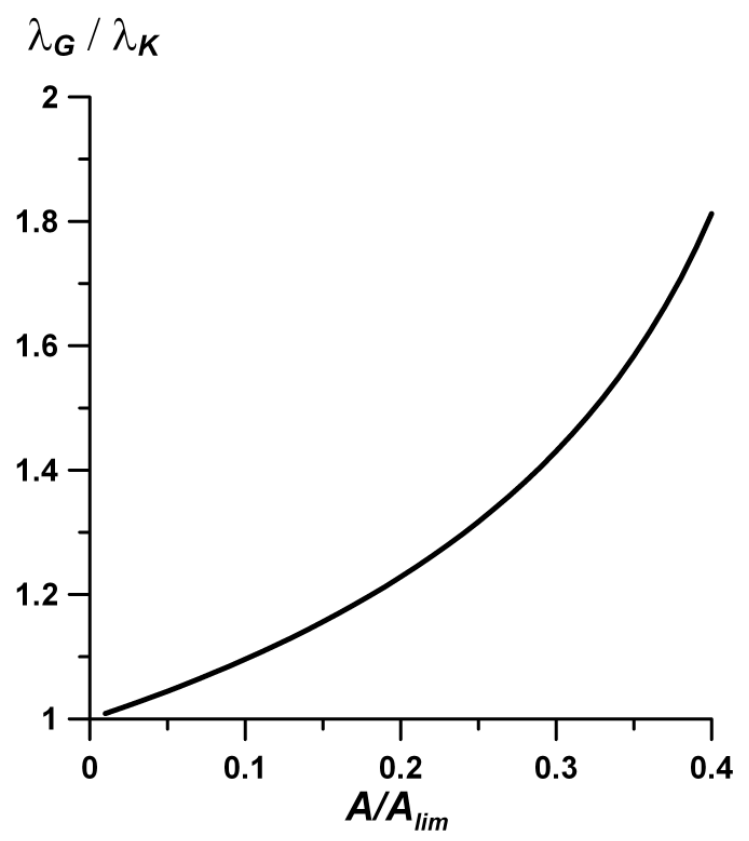

Fig. 2. The ratio of the Gardner soliton length to the KdV soliton length versus $A / A_{\text {lim }}$.

waves the quadratic nonlinear term prevails. With increase in the wave amplitude the cubic nonlinear term is comparable with the quadratic one, and in the case of the limiting soliton having amplitude Eq. (16) it is fully compensated, so that the nonlinear parameter is $\varepsilon_{\lim }=0$. The maximum of $\varepsilon$ is for $A=A_{\mathrm{lim}} / 2$. In fact, the parameter $\varepsilon$ characterizes the nonlinear correction to the soliton speed, and solitons of small and large amplitudes (with respect to the limiting values) move with the same speed. Alternatively nonlinearity can be characterized by

$\varepsilon_{n}=\varepsilon_{q}+\left|\varepsilon_{c}\right|=\frac{\alpha A}{c}+\frac{\left|\alpha_{1}\right| A^{2}}{c}$.

With this definition the nonlinear parameter is maximal for the soliton of limiting amplitude. The influence of the cubic nonlinearity on the soliton characteristics can be shown by comparing the Gardner soliton length $\lambda_{G}$ and the KdV soliton length $\lambda_{K}$; their ratio depends on the ratio of the soliton amplitude $A$ to the table-top soliton amplitude $A_{\text {lim }}$ Eq. (16),

$\frac{\lambda_{G}}{\lambda_{K}}=\sqrt{\frac{A_{\lim }}{A}} \tanh ^{-1}\left[\sqrt{\frac{1}{\frac{A_{\lim }}{A}-1}}\right]$.

Here both soliton lengths are defined as the soliton mass (defined here as the integral of $\eta$ over all $x$ ) divided by the soliton amplitude (Grimshaw et al., 2002a). This ratio is shown in Fig. 2. Note that when the ratio $A / A_{\lim }$ is 0.2 the difference in the lengths of the Gardner and $\mathrm{KdV}$ solitons is $20 \%$, but when the ratio $A / A_{\lim }=0.3$ it is $40 \%$. Based on Fig. 2, we call waves with $A / A_{\lim }<0.1$ weakly nonlinear (KdV waves).

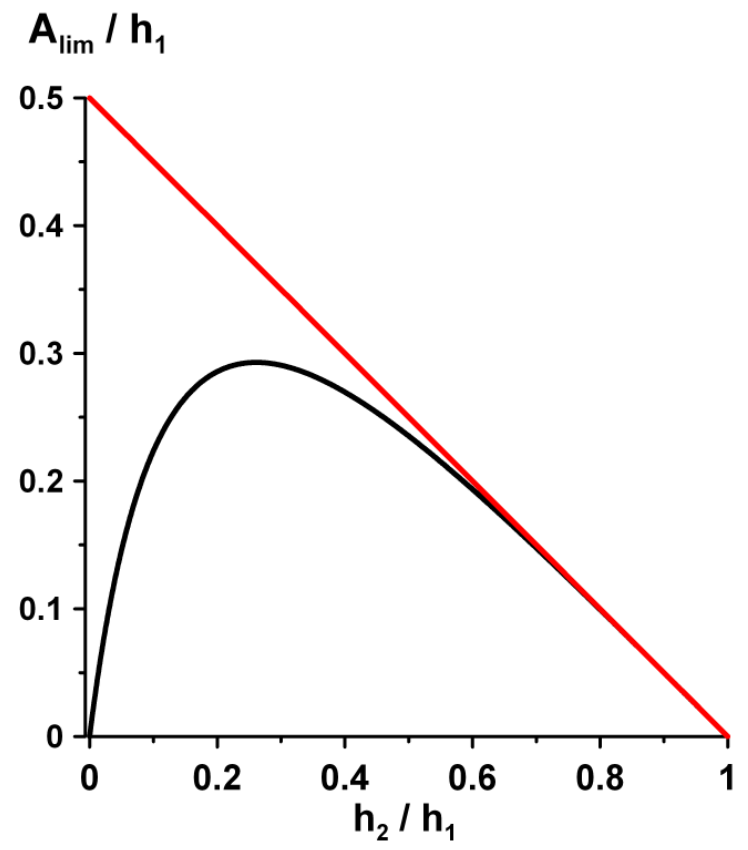

Fig. 3. The limiting soliton amplitude versus the thicknesses of the layers: the red line is the Euler soliton amplitude and the black line is the Gardner soliton amplitude.

Waves with $A / A_{\text {lim }}>0.1$ require another classification. If $\varepsilon_{n}$ is relatively small, the Gardner equation is valid because it is derived formally using a perturbation technique based on the small nonlinear parameter $\varepsilon$ up to the second order. We may call such waves Gardner waves, or moderate nonlinear waves. If $\varepsilon_{n}$ is comparable with 1 or exceeds it, the Gardner equation is formally not applicable, and such waves are strongly nonlinear. The applicability of the Gardner equation can be analyzed by comparison of the limiting solitary waves in the full Euler and the Gardner equations. Solitary waves in the Euler equations have been studied intensively (Grue et al., 1999; Choi and Camassa, 1999); they also exist for amplitudes less than the maximal value

$\widehat{A}_{\lim }=\frac{h_{1}-h_{2}}{2}$

Comparison of the limiting soliton amplitudes in the Gardner and Euler equations is shown in Fig. 3. The curves are close to each other if $0.6<h_{2} / h_{1}<1$, which corresponds to wave amplitudes greater than $0.2 h_{1}$ or $h_{2} / 3$. This value separates the regimes of moderate and strongly nonlinear waves. The formulas given above will be used for comparison with the results from the fully nonlinear simulations.

\section{Numerical fully nonlinear model}

The numerical model is based on the Navier-Stokes equations for a continuously stratified fluid (Kanarska and 
Maderich, 2003; Brovchenko et al., 2007). It is a nonhydrostatic extension of the Princeton Ocean Model (POM). The density stratification in the numerical simulations is modeled by salinity stratification, appropriate for a typical laboratory experiment. The basic equations for continuity, momentum and salinity are written in the Boussinesq approximation as,

$\nabla \cdot \boldsymbol{U}=0$

$\frac{D \boldsymbol{U}}{D t}=-\frac{1}{\rho_{0}} \nabla P+v \nabla^{2} \boldsymbol{U}-\boldsymbol{g} \frac{\rho}{\rho_{0}}$,

$\frac{D S}{D t}=\chi \nabla^{2} S$.

Here $\boldsymbol{U}=(U, V, W)$ is in general the three-dimensional velocity in Cartesian coordinates $\boldsymbol{x}=(x, y, z)$, with $x$ a coordinate along the computational flume, $y$ is the transverse coordinate, and $z$ is directed vertically upward; $D / D t$ is the material derivative; $P$ is the pressure; $\rho$ is density and $\rho_{0}$ is the undisturbed density; $\boldsymbol{g}=(0,0, g), g$ is the gravitational acceleration; $S$ is a salinity; $v$ and $\chi$ are the kinematic viscosity and diffusivity respectively. The system Eqs. (23)-(25) is closed by an equation of state (Mellor, 1991) for the density $\rho$ of water $\rho=\rho(S, T, P)$. The numerical solution of these governing equations, with the relevant boundary conditions on the solid boundaries and the free-surface, is based on the modified algorithm by Kanarska and Maderich (2003) with a four-stage procedure; (i) computation of the free surface level and the depth-integrated velocity field; (ii) computation of the provisional hydrostatic components of velocity; (iii) computation of the non-hydrostatic components of the velocity and pressure fields; (iv) computation of the scalar fields. The computational tank has the geometry shown in Fig. 1. The total length of the flume is $24 \mathrm{~m}$, with the length of the deep part $L_{1}=10 \mathrm{~m}$. The problem is solved in a twodimensional mode. Non-slip boundary conditions $(U=0)$ at the bottom and end walls were used, whereas at the free surface the viscous stresses were set to zero. The flux of salinity through the flume boundaries was set to zero.

The background stratification in the flume is modelled by two layers with upper and bottom layer salinities $S_{\text {up }}=2$ and $S_{\text {bot }}=15$ at constant temperature, respectively. The density jump $\Delta \rho / \rho_{0}$ is equal 0.01 . The vertical profile $S(z)$ in the transition zone is approximated by

$S(z)=\frac{S_{\mathrm{up}}+S_{\mathrm{bot}}}{2}-\frac{S_{\mathrm{up}}-S_{\mathrm{bot}}}{2} \tanh \left(\frac{\left(z-h_{1}\right)}{d h}\right)$

where $d h=0.2 \mathrm{~cm}$ is much less than the thickness of the layers. In the simulations we visualized the interface as an isocline with salinity equal 8.5. Numerical experiments were carried out with molecular values of viscosity $\nu=0.01 \mathrm{~cm}^{2} \mathrm{~s}^{-1}$ and diffusivity of salt $\chi=1 \cdot 10^{-3} \mathrm{~cm}^{2} \mathrm{~s}^{-1}$. The computational grid was $1900 \times 120$.
Table 1. Gardner equation parameters.

\begin{tabular}{lll}
\hline & $\begin{array}{l}\text { Right from } \\
\text { a step }(-)\end{array}$ & $\begin{array}{l}\text { Left from } \\
\text { a step }(+)\end{array}$ \\
\hline$c, \mathrm{~cm} / \mathrm{s}$ & 7.48 & 5.71 \\
$\alpha, \mathrm{s}^{-1}$ & 0.842 & 1.715 \\
$\alpha_{1}, \mathrm{~cm}^{-1} * \mathrm{~s}^{-1}$ & -0.156 & -0.3 \\
$\beta, \mathrm{cm}^{3} / \mathrm{s}$ & 199.55 & 76.21 \\
$A_{\text {lim }}, \mathrm{cm}$ (Gardner) & 5.4 & 5.7 \\
$\hat{A}_{\text {lim }} \mathrm{cm}$ (Navier-Stokes) & 6 & 8 \\
\hline
\end{tabular}

The problem of initialization for the Navier-Stokes equations is not simple for large amplitude internal solitary waves in a continuously stratified fluid because we are required to specify two-dimensional salinity and velocity fields. Vlasenko and Hutter (2001) used for initialization the analytical solution that describes a weakly nonlinear KdV solitary wave. Often the solitary wave in a laboratory tank is generated by a "collapse" mechanism (see e.g. Chen et al., 2007). To generate the leading wave of elevation, the initial thickness of the collapsing volume should be less than the thickness of the upper layer; in the opposite case a depression wave is generated. In our simulations this approach is applied and a leading wave of elevation type is generated. Then the tail of small scale waves was cut out.

In the numerical experiments the thickness of the upper layer is $h_{1}=20 \mathrm{~cm}$. The thickness of the lower layer is $h_{-}=8 \mathrm{~cm}$ (on the right before the step) and $h_{+}=4 \mathrm{~cm}$. Initial wave amplitudes are $1 \mathrm{~cm}$ and $4 \mathrm{~cm}$. For these conditions the calculated coefficients of the Gardner equation are given in Table 1 . The amplitudes of the limiting solitary waves computed in the framework of the Gardner and Navier-Stokes equations are also presented. In the experiment the thickness of the layers differ significantly $\left(h_{2} / h_{1}=0.2-0.4\right)$ and formally the limiting wave is a strongly nonlinear wave. In fact, the limiting solitary wave is more nonlinear after the step than before, and this is manifested in the difference between the amplitude values in Table 1. So, in our experiments formally we could not use the Gardner equation for the description of the limiting solitary waves.

For an initial wave of $1 \mathrm{~cm}$ the cubic nonlinear term is small, and the nonlinear parameter $\varepsilon \sim \varepsilon_{n} \sim 0.1$, but $A / A_{\lim }>0.1$. Therefore, the initial solitary wave is a moderate nonlinear wave which can be described by the Gardner equation. The soliton with initial amplitude $4 \mathrm{~cm}$ is strongly nonlinear, because $\varepsilon=0.12$ and $\varepsilon_{n}=0.78$. Formally, the Gardner equation is not applicable for this case. Analysis of the wave dynamics in both cases using the fully nonlinear and the Gardner equations can clarify the applicability of these approximate models to describe the solitary waves of different amplitudes. 
$\eta, \mathbf{c m}$

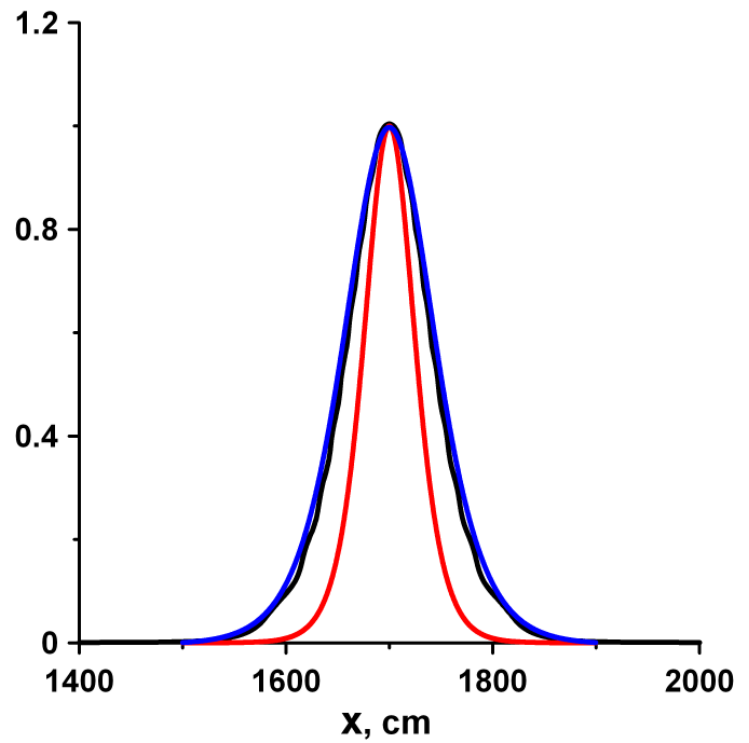

Fig. 4. The initial pulse (black) and its approximation by a KdV soliton (red) and Gardner soliton (blue).

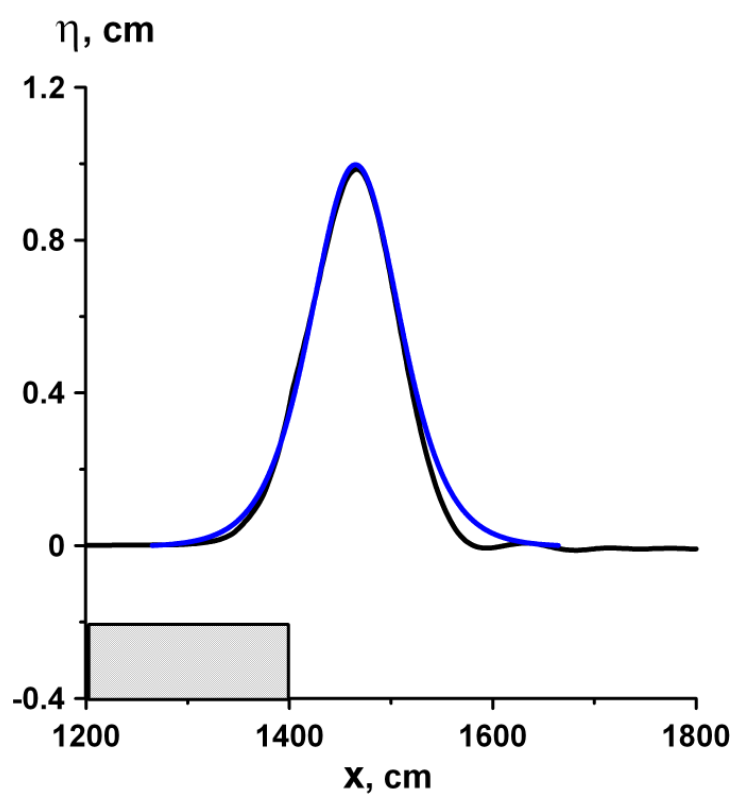

Fig. 5. The wave approaching the step ( $t=45 \mathrm{~s})$ from the right (black) and the Gardner soliton approximation (blue).

\section{Simulation of the transformation of a moderate am- plitude wave}

The first simulation is for an initial moderate solitary wave of $1 \mathrm{~cm}$ amplitude. According to the linear theory Eq. (1) the reflected wave has an amplitude $0.1 \mathrm{~cm}$ and is very weak. The transmitted wave is about $1.3 \mathrm{~cm}$ in amplitude, which is com-

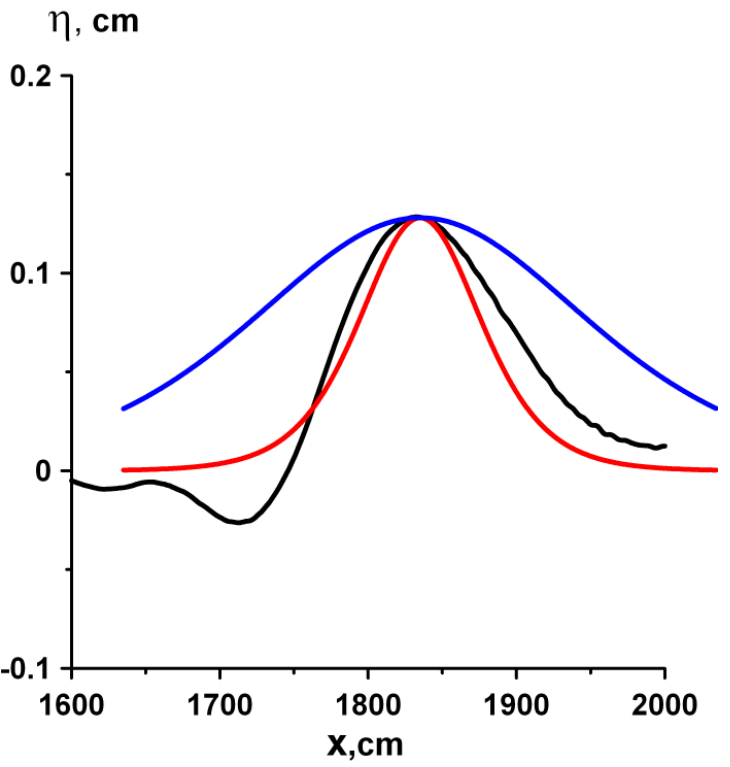

Fig. 6. The reflected wave (black) near the tank wall, and the comparison with a KdV (red) and Gardner (blue) soliton shapes.

parable with the thickness of lower layer after the step $(4 \mathrm{~cm})$. Hence, nonlinear effects in the transmitted wave should be essential. According to the KdV prediction (2.11) three solitons should be formed far from a step. As we have indicated, the wave has a moderate amplitude and we should instead apply the Gardner equation. The initial impulse generated by the collapse mechanism is displayed in Fig. 4. The theoretical shapes of the KdV (red) and Gardner (blue) solitons are also presented. The agreement with the Gardner soliton shape is excellent, but the KdV soliton is narrower than the observed soliton. The soliton approaches the step (from right to left) and keeps its shape very well (Fig. 5).

The reflected wave has a very small amplitude (about $1.3 \mathrm{~mm}$ ), and moves with a very slow change in shape and amplitude. This process is unsteady, and the wave shape is not well described by the KdV or Gardner soliton shapes (see Fig. 6). The wave transformation at the step is illustrated in Fig. 7. It is seen that transmitted wave has increased in amplitude up to $1.1 \mathrm{~cm}$ and propagates into shallow water. A weak wave of $0.13 \mathrm{~cm}$ amplitude reflects from the step. The computed wave amplitudes in the vicinity of a step are in good agreement with the predictions of linear theory Eq. (1). Far from the step (225 s after generation) the transmitted wave transforms into two soliton-like waves. The shape of the leading wave is quite well described by both the $\mathrm{KdV}$ and Gardner equations (Fig. 8), because its amplitude $1.4 \mathrm{~cm}$ is less then maximal value for the soliton amplitude $(6-8 \mathrm{~cm})$.

The process of soliton fission is not finished at this time. According to KdV theory, the amplitude of the leading soliton at the final stage should be $1.5 \mathrm{~cm}$. To compare the results of the fully nonlinear simulations with the theoretical 

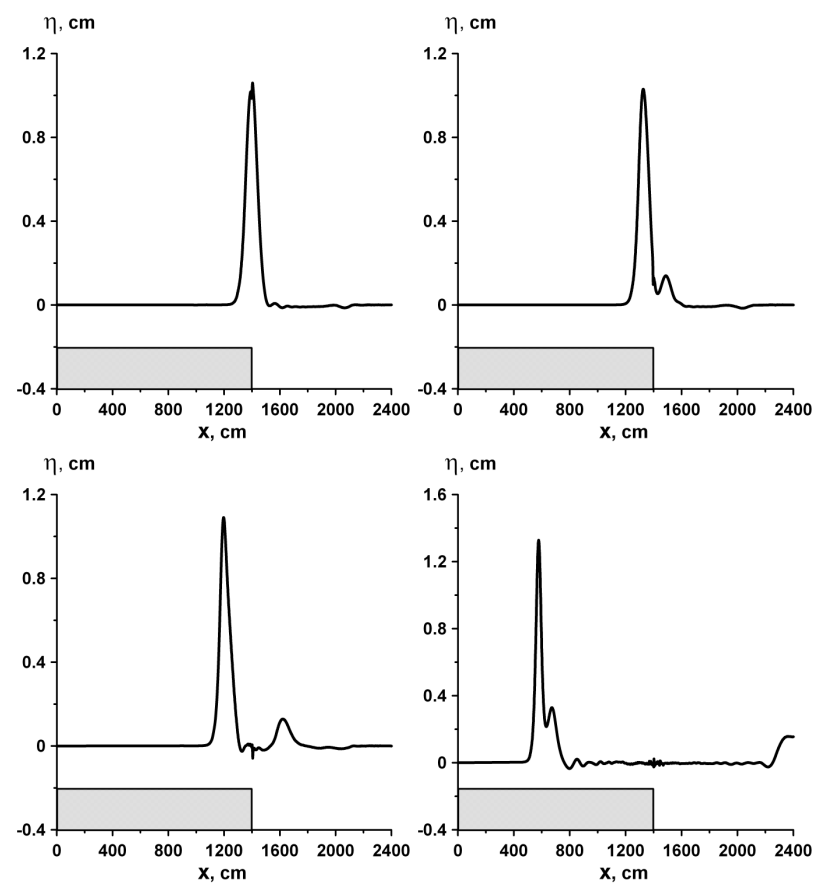

Fig. 7. Wave transformation at the step.

predictions, a numerical simulation was also performed in the framework of the Gardner equation for the wave propagation after the step with the initial condition Eq. (18). A finite-difference scheme which satisfies the Courant criterion is used to solve the Gardner equation (Holloway et al., 1997, 1999). The comparison between the Gardner (blue) and full nonlinear (black) computations is presented on Fig. 9.

The wave shape just after the step is almost the same in the Gardner and Navier-Stokes simulations (Fig. 9a), and the difference in amplitudes is $0.09 \mathrm{~cm}$ or $8 \%$. Far from the step there is relatively large difference in the leading soliton amplitudes at the time $t=225 \mathrm{~s}$, and in their locations (Fig. 9b). The amplitude of the first soliton far from the step in the Gardner model is $1.5 \mathrm{~cm}$ and in the full equation model it is $1.35 \mathrm{~cm}$, a difference of about $10 \%$. This difference in wave amplitudes is related with the difference in the wave amplitudes just after the step, and the difference in the numerical models. The big difference in the wave locations, $34 \mathrm{~cm}$ (Fig. 9b) can be explained by the difference in the wave speed $(0.26 \mathrm{~cm} / \mathrm{s}$ or $5 \%$ of mean nonlinear velocity), as even if the amplitudes are close together, at large times (distances) the phases will be significantly different.

So, the case of a moderate nonlinear interfacial solitary wave interaction with a bottom step shows good applicability of the weakly nonlinear theory for the description of the transmitted and reflected wave shapes and their amplitudes (the difference is 10\%).

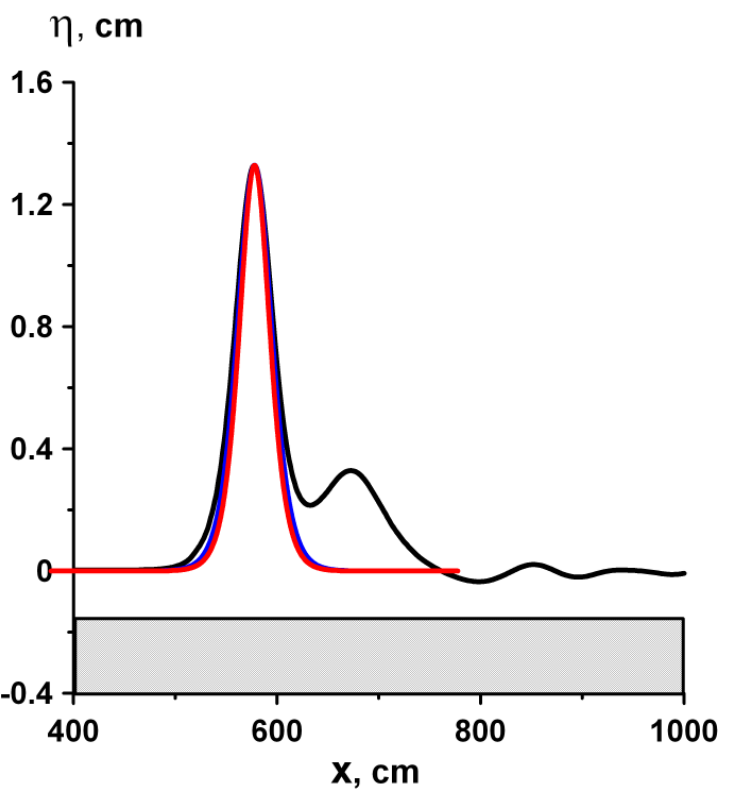

Fig. 8. Formation of secondary solitons in the transmitted wave (experimental wave shape - black) and comparison of the leading wave with a KdV soliton (red) and Gardner soliton (blue).
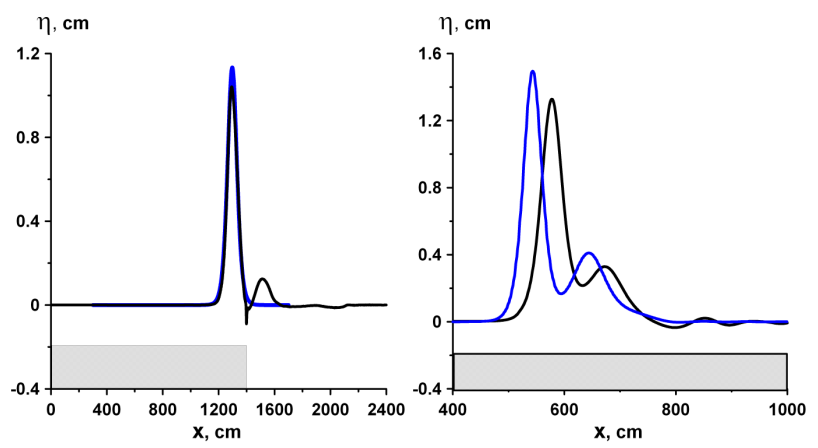

Fig. 9. Comparison of Gardner (blue line) and Navier-Stokes (black line) modeling.

\section{Large-amplitude wave transformation at a step}

The next simulation is for an initial solitary wave amplitude of $4 \mathrm{~cm}$. As already indicated, in this case the solitary wave can be considered as strongly nonlinear. The incident wave is shown in Fig. 10 for two time in comparison with the Gardner soliton shape. It is evident that the Gardner and NavierStokes wave shapes coincide quite well. Due to strong nonlinearity the incident wave "feels" the step through its tail and the radiation of a dispersive wavetrain begins immediately. A "shelf" of opposite sign is generated behind the solitary wave approaching the step (Fig. 10b). The total wave amplitude is increased due to the interaction with the reflected wave from $4 \mathrm{~cm}$ to $4.27 \mathrm{~cm}$. 

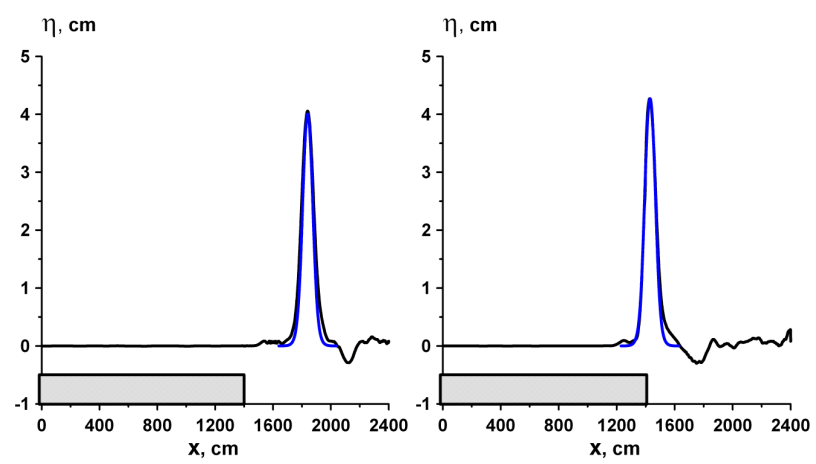

Fig. 10. Incident wave (black) and its Gardner soliton approximation (blue).

The wave transformation after passage over the step is illustrated by snapshots shown in Fig. 11. It is interesting to note that dividing the pulse into the transmitted and reflected waves occurs at the time when the peak is just before the step (Fig. 11a), which has also been noted for the weak nonlinear case (see Fig. 6a). The wave amplitude here is $4.61 \mathrm{~cm}$ and begin to decrease as the wave crosses the step. At $t=80 \mathrm{~s}$ (Fig. 11b) when the wave peak has just crossed the step (Fig. 11b) its amplitude is $3.93 \mathrm{~cm}$, but there is no full separation on the transmitted and reflected waves. The division into transmitted and reflected wave is almost completed at $t=89 \mathrm{~s}$ (Fig. 11c). The leading wave amplitude is increasing now to $4.33 \mathrm{~cm}$, mainly due to the beginning of secondary solitary wave generation.

The generation of secondary solitary waves (fission) begins in the transmitted wave just after the step. The wave amplitude grows (Fig. 11d, c) and the wave transforms into solitary waves (Fig. 11e, f), the leading wave has an amplitude of $5.4 \mathrm{~cm}$. It is a bit less than the table-top solitary wave amplitude of $5.7 \mathrm{~cm}$ (according to the Gardner theory). The second solitary wave has an amplitude of $1.8 \mathrm{~cm}$ and third one $0.54 \mathrm{~cm}$. Figure 12 presents a zoom of both generated solitary waves from Fig. 11f in comparison with the Gardner equation modeling of the transmitted wave. The leading wave in the full nonlinear computations is close to the Gardner soliton, but the Gardner soliton seems to be a little bit wider and its amplitude is somewhat less $(5.2 \mathrm{~cm})$. The difference in amplitudes of the leading solitary waves is $0.2 \mathrm{~cm}$, (about 4\%). The second Gardner soliton has an amplitude of $0.96 \mathrm{~cm}$ and it is about half the fully-modeled second solitary wave with an amplitude of $1.8 \mathrm{~cm}$. The third soliton in the Gardner modeling has amplitude of $0.21 \mathrm{~cm}$ and it is also more than half the fully-modeled third solitary wave. Nevertheless, this last soliton in the Gardner model isn't formed completely at this time. For comparison we also give the predictions of the $\mathrm{KdV}$ theory for secondary soliton amplitudes: $6.2 \mathrm{~cm}$ for the leading soliton (about $20 \%$ more than Gardner and fully-modeled waves), $1.8 \mathrm{~cm}$ for the second soliton (double the second Gardner wave and equal to the full-
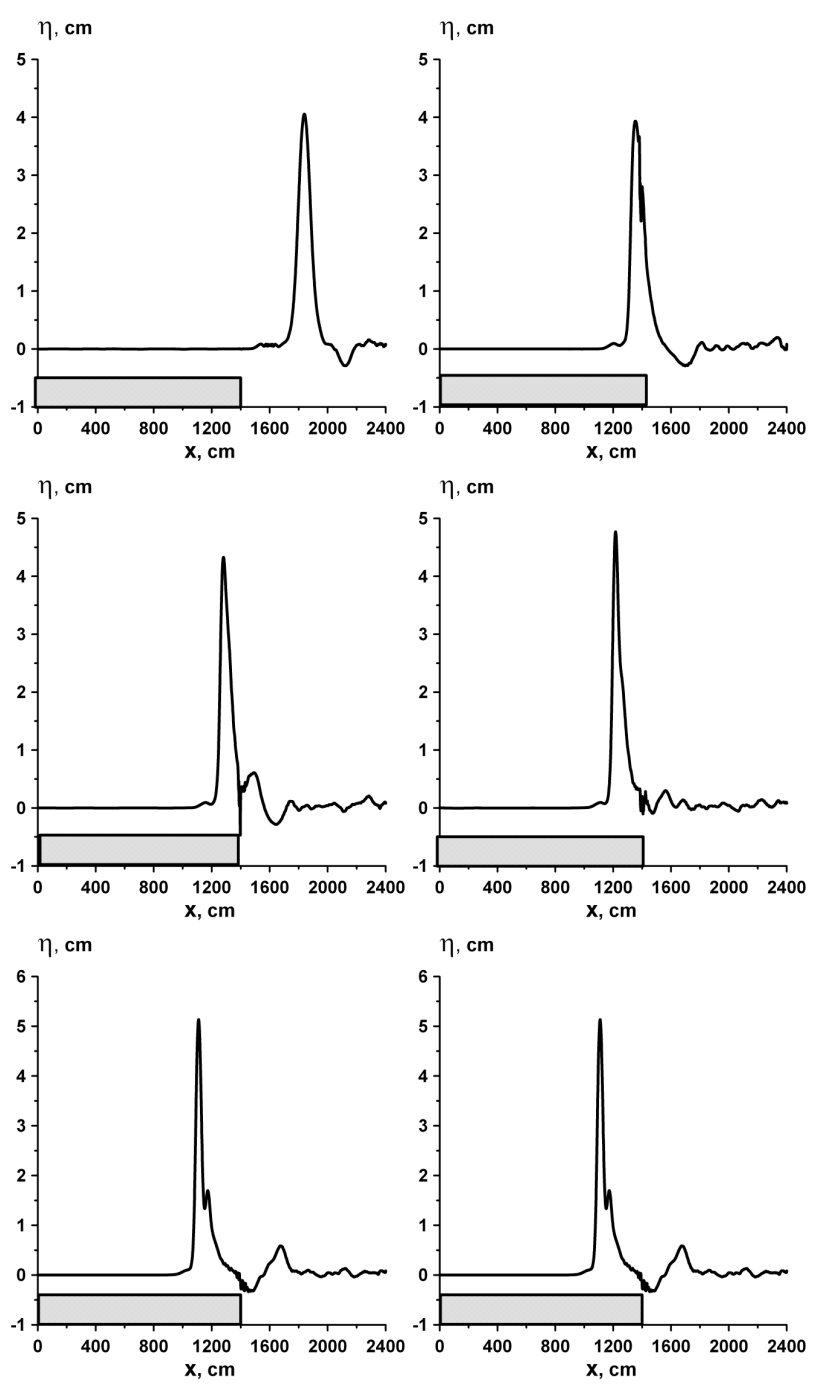

Fig. 11. Large-amplitude wave formation at a step.

modeled waves!) and $0.05 \mathrm{~cm}$ for the third soliton, that is 10 times less than the fully-modeled solitary wave.

The shift in positions of the leading solitary waves on Fig. 12 is about $33 \mathrm{~cm}$ and it is only $2 \%$ of the distance which both solitary waves have gone from the step. This takes a time of about $190 \mathrm{~s}$, and the difference in the velocities of both nonlinear waves is about $0.2 \mathrm{~cm} / \mathrm{s}(3 \%$ of the mean nonlinear velocity), and the Gardner solitary wave is faster than the fully-modeled one. The difference in velocities for the second solitary waves is smaller, $0.06 \mathrm{~cm} / \mathrm{s}$ (shift is $12 \mathrm{~cm}$ ).

The reflected wave has amplitude of about $0.56 \mathrm{~cm}$, which is close to the linear prediction $(0.54 \mathrm{~cm})$. It is shown in Fig. 13 in comparison with the KdV (red) and Gardner (blue) solitons. It seems that a solitary wave has not formed at this time.

Hence, in this strongly nonlinear case we also have a reasonably good comparison of the fully nonlinear simulations 


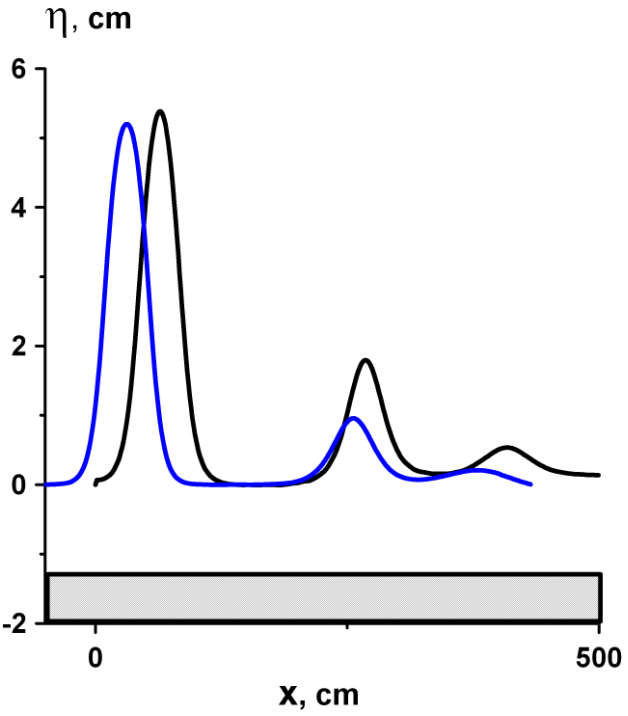

Fig. 12. Comparison of models after step, $t=259 \mathrm{~s}$. Navier-Stokes model is black line, the Gardner model is blue line.

with the predictions of the theoretical model based on the Gardner equation. The difference in the leading soliton amplitude in the fully nonlinear model and the Gardner simulations is about $4 \%$, and about $2 \%$ in velocity. The difference in amplitudes of the following secondary solitary waves in both models is more significant, and it reaches to $50 \%$.

\section{Conclusions}

We have studied the transformation of an interfacial solitary wave transformation at a bottom step numerically in the framework of the fully nonlinear equations. The wave transformation in the vicinity of the step is reasonably well described by linear long wave theory (the difference in the predictions for the amplitudes of the transmitted and reflected waves is less $10 \%$ ) for moderate nonlinear waves and for strongly nonlinear waves. Indeed, in this respect our simulations coincide with conclusions made previously for surface waves over a bottom step. The process of solitary wave fission in the transmitted wave is simulated in both the fully nonlinear model and in the Gardner equation. This last equation includes an additional cubic nonlinear term into the usual KdV equation, and can be applied for interfacial waves of weak and moderate amplitudes. The shapes of the computed solitary waves are well described by the soliton solution of the Gardner equation. Although it is not expected that the Gardner model should describe the properties of strongly nonlinear waves with good accuracy, in reality, and to our surprise, the Gardner model is appropriate for the leading solitary in the strongly nonlinear case. For instance, the difference in its amplitude in the fully nonlinear model and the Gardner simulations is about $4 \%$, compared with $10 \%$ for

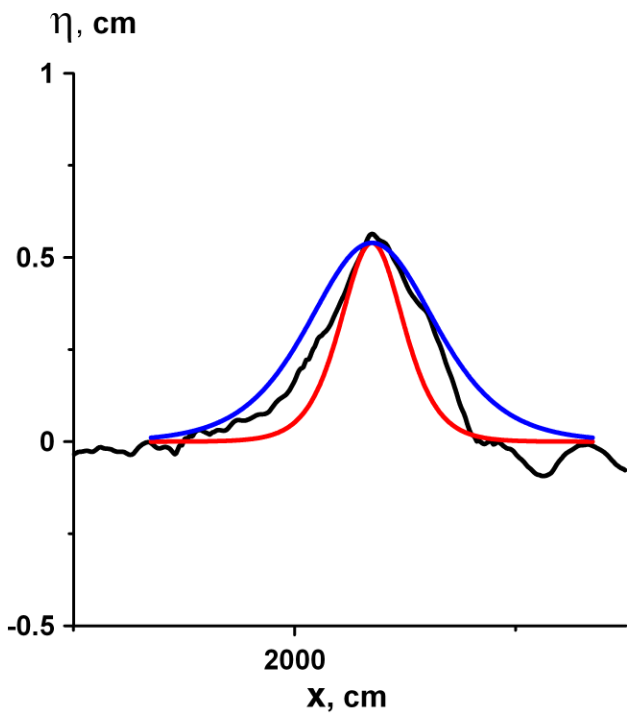

Fig. 13. Reflected wave (zoom). The fully nonlinear model is the black line, the Gardner model is the blue line, and the KdV model is the red line.

the case of moderate amplitudes. It is also interesting to note that the strongly nonlinear solitary wave has a larger amplitude then the Gardner soliton; in the moderate nonlinear case the situation is opposite. The difference in velocities of the solitary wave propagation is about $2 \%$ for strongly nonlinear waves, but for moderate waves this difference is $5 \%$. The difference in amplitudes of the following secondary solitary waves between both models reaches to $50 \%$ in strong nonlinear case. Nevertheless it seems that the Gardner model can better describe the strongly nonlinear case than the moderate nonlinear case, at least for the leading wave.

Finally we note that although our fully nonlinear numerical simulations were carried out for a typical laboratory configuration, the results can be readily extrapolated to an oceanic situation by simply rescaling the length and time scales. When this is done, the main issue of concern would be how well the kinematic viscosity and diffusivity would scale into the oceanic situation. This is unknown, but we note that the good agreement here with the Gardner and KdV models, which contain no such entities, suggests that the results obtained here are not dependant on the kinematic viscosity and diffusivity in any essential way.

Acknowledgements. The authors thank the support of UK-Russian grant RFBR 08-05-91850-KO (T. T., E. P., R. G.), RussianUkrainian grant RFBR 09-05-90408 (V. M., T. T., I. B., K. T.), RFBR 09-05-00204 (T. T.), Leverhulme Trust (E. P.), and BK21 (B. H. Choi).

Edited by: I. Didenkulova

Reviewed by: Y. Stepanyants and N. Makarenko 


\section{References}

Bogucki, D. and Redekopp, L.: A mechanism for sediment resuspension by internal solitary waves, Geophys. Res. Lett., 26, 9, 1317-1320, 1999.

Bourgault, D., Blokhina, M. D., Mirshak, R., and Kelley, D. E.: Evolution of a shoaling internal solitary wavetrain, Geophys. Res. Lett., 34, L03601, doi:10.1029/2006GL028462, 2007.

Brovchenko, I., Gorodetska, N., Maderich, V., Nikishov, V., and Terletska, K.: Interaction of internal solitary waves of large amplitude with obstacle, Applied Hydromechanics, 9(81), No. 1, 3-7, 2007

Chang, K.-A., Hsu, T.-J., and Liu, P. L.-F.: Vortex generation and evolution in water waves propagating over a submerged rectangular obstacle, Coastal Eng., 44, 13-36, 2001.

Chen, C.-Y., Hsu, J. R.-C., Chen, H.-H., Kuo, C.-F., and Cheng, M.H.: Laboratory observations on internal solitary wave evolution on steep and inverse uniform slopes, Ocean Eng., 34, 157-170, 2007a.

Chen, C.-Y., Hsu, J. R.-C., Cheng-Wu Chen, C.-W., Chen, H.-H., Kuo, C.-F., and Cheng, M.-H.: Generation of internal solitary wave by gravity collapse, J. Mar. Sci. Technol., 15, 1-7, 2007 b.

Chen, C.-Y., Hsu, J. R.-C., Cheng, M.-H., Chen, H.-H., and Kuo, C.-F.: An investigation on internal solitary waves in a two-layer fluid: Propagation and reflection from steep slopes, Ocean Eng., 34, 171-184, 2007c.

Choi, W. and Camassa, R.: Fully nonlinear internal waves in a twofluid system, J. Fluid Mech., 396, 1-36, 1999.

De Zárate, A. R. and Nashbin, A.: A reduced model for internal waves interacting with topography at intermediate depth, Commun. Math. Sci., 6, 385-396, 2008.

Djordjevic, V. and Redekopp, L.: The fission and disintegration of internal solitary waves moving over two-dimensional topography, J. Phys. Ocean., 8, 1016-1024, 1978.

Grimshaw, R., Pelinovsky, D., Pelinovsky, E., and Slunyaev, A.: The generation of large- amplitude solitons from an initial disturbance in the extended Korteweg - de Vries equation, Chaos, 12, 1070-1076, 2002a.

Grimshaw, R., Pelinovsky, E., and Poloukhina, O.: Higher-order Korteweg-de Vries models for internal solitary waves in a stratified shear flow with a free surface, Nonlin. Processes Geophys., 9, 221-235, 2002b,

http://www.nonlin-processes-geophys.net/9/221/2002/.

Grimshaw, R., Pelinovsky, E., Talipova, T., and Kurkin, A.: Simulation of the transformation of internal solitary waves on oceanic shelves, J. Physical Oceanography, 34, 2774-2779, 2004.

Grimshaw, R., Pelinovsky, E., and Talipova, T.: Modeling internal solitary waves in the coastal ocean, Survey in Geophysics, 28, 273-298, 2007

Grimshaw, R., Pelinovsky, E., and Talipova T.: Fission of a weakly nonlinear interfacial solitary wave at a step, Geophysical and Astrophysical Fluid Dynamics, 102, 179-194, 2008.

Grue, J., Jensen, A., Rusås, P.-O., and Sveen, J. K.: Properties of large amplitude internal waves, J. Fluid Mech., 380, 257-278, 1999.

Helfrich, K. R.: Internal solitary wave breaking and run-up on a uniform slope, J. Fluid Mech., 243, 133-154, 1992.
Helfrich, K. R. and Melville, W. K.: On long nonlinear internal waves over slope-shelf topography, J. Fluid Mech., 167, 285308, 1986.

Holloway, P., Pelinovsky, E., Talipova, T., and Barnes, B.: A Nonlinear Model of Internal Tide Transformation on the Australian North West Shelf, J. Physical Oceanography, 27, 871-896, 1997.

Holloway, P., Pelinovsky, E., and Talipova, T.: A Generalized Korteweg - de Vries Model of Internal Tide Transformation in the Coastal Zone, J. Geophys. Res., 104(C8), 18333-18350, 1999

Kakutani, T. and Yamasaki, N. Solitary waves on a two-layer fluid, J. Phys. Soc., Japan, 45, 674-679, 1978.

Kanarska, Y. and Maderich, V.: A non-hydrostatic numerical model for calculating of free-surface stratified flows, Ocean Dynam., $51,176-185,2003$.

Lamb, K. G.: A numerical investigation of solitary internal waves with trapped cores formed via shoaling, J. Fluid Mech., 451, 109-144, 2002.

Lamb, K. G.: Shoaling solitary internal waves: on a criterion for the formation of waves with trapped cores, J. Fluid Mech., 478, 81-100, 2003.

Lin, P.: A numerical study of solitary wave interaction with rectangular obstacles, Coastal Eng., 51, 35-51, 2004

Liu, P. L.-F. and Cheng, Y.: A numerical study of the evolution of a solitary wave over a shelf, Physics Fluids, 13, 1660-1667, 2001.

Mellor, G. L.: An equation of state for numerical models of ocean and estuaries, J. Atmos. Ocean. Tech., 8, 609-611, 1991.

Orr, M. H. and Mignerey, P.C.: Nonlinear internal waves in the South China Sea: observation of the conversion of depression internal waves to elevation internal waves, J. Geophys. Res., 108(C3), 3064, doi:10.1029/2001JC001163, 2003.

Ramp, S. R., Tang, T. Y., Duda, T. F., Lynch, J. F., Liu, A. K., Chiu, C.-S., Bahr, F. L., Kim, H.-R., and Yang, Y.-J.: Internal Solitons in the Northeastern South China Sea Part I: Sources and Deep Water Propagation, IEEE J. Oceanic Eng., 29, 1157-1181, 2004.

Ribbe, J. and Holloway, P.: A model of suspended sediment transport by internal tides, Cont. Shelf Res., 21, 395-422, 2001.

Seabra-Santos, F. J., Renouard, D. P., and Temperville, A. M.: Numerical and experimental study of the transformation of a solitary wave over a shelf or isolated obstacle, J. Fluid Mech., 176, 117-134, 1987.

Stastna, M. and Lamb, K. G.: Sediment resuspension mechanisms associated with internal waves in coastal waters, J. Geophys. Res., 113, C10016, doi:10.1029/2007JC004711, 2008.

Vlasenko, V. I. and Hutter, K.: Generation of second mode solitary waves by the interaction of a first mode soliton with a sill, Nonlin. Processes Geophys., 8, 223-239, 2001, http://www.nonlin-processes-geophys.net/8/223/2001/.

Vlasenko, V. and Stashchuk, N.: Three-dimensional shoaling of large-amplitude internal waves, J. Geophys. Res., 112, C11018, doi:10.1029/2007JC004107, 2007.

Vlasenko, V., Stashchuk, N., and Hutter, K.: Baroclinic tides: theoretical modeling and observational evidence, Cambridge Univ. Press, 2005.

Zhao, Z., Klemas, V. V., Zheng, Q., and Yan, X.-H.: Satellite observation of internal solitary waves converting polarity, Geophys. Res. Lett., 30(19), 1988, doi:10.1029/2003GL018286, 2003.

Zheng, Q., Klemas, Q., Yan, X-H., and Pan, J.: Nonlinear evolution of ocean internal solitons propagating along an inhomogeneous thermocline, J. Geophys. Res., 106(C7), 14083-14094, 2001. 